\title{
Glioma cells enhance endothelial progenitor cell angiogenesis via VEGFR-2, not VEGFR-1
}

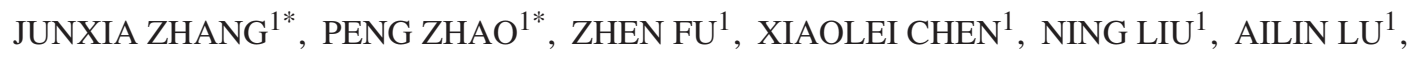 \\ RUI LI ${ }^{1}$, LEI SHI ${ }^{1}$, PEIYU PU ${ }^{2}$, CHUNSHENG KANG ${ }^{2}$ and YONGPING YOU ${ }^{1}$ \\ ${ }^{1}$ Department of Neurosurgery, the First Affiliated Hospital of Nanjing Medical University, Nanjing 210029; \\ ${ }^{2}$ Department of Neurosurgery, Tianjin Medical University General Hospital, Tianjin 300052, P.R. China
}

Received June 10, 2008; Accepted August 29, 2008

DOI: 10.3892/or_00000166

\begin{abstract}
Although potential contribution of endothelial progenitor cells (EPCs) to angiogenesis in glioma has been proposed, the molecular mechanisms of EPCs recruitment to vasculature have not been fully elucidated. Here, we show that the supernatant from glioma cells promotes EPCs angiogenesis via VEGFR-2, not VEGFR-1. Moreover, VEGFR-2 siRNA inhibits VEGFR-2 expression in EPCs, tube formation on matrigel and cell migration. MMP-9 activity and expression and the Akt and ERK phosphorylations are decreased by VEGFR-2 siRNA. Thus, these results indicate that glioma cells enhance EPC angiogenesis via VEGFR-2, not VEGFR-1, mediated by the MMP-9, Akt and ERK signal pathways.
\end{abstract}

\section{Introduction}

Glioma remains one of the human tumors most refractory to treatment, despite continuing advances in radiotherapy, chemotherapy, and surgical techniques. The treatment failure is due to diffuse infiltration of the surrounding 'normal brain tissues'. The current wisdom is that tumor growth, invasion and metastasis are angiogenesis-dependent. Glioma is one of the best vascularized tumors and its proliferation is hallmarked by a distinct proliferative vascular component. Therefore, developing angiogenesis-targeted therapeutic strategies for glioma would be a top priority.

Recent studies have provided ample evidence that angiogenesis is required for the mobilization and functional incorporation of endothelial progenitor cells (EPCs). The

Correspondence to: Dr Yongping You, Department of Neurosurgery, the First Affiliated Hospital of Nanjing Medical University, Nanjing 210029, P.R. China

E-mail: yyp19@njmu.edu.cn

Dr Chunsheng Kang, Department of Neurosurgery, Tianjin Medical University General Hospital, Tianjin 300052, P.R. China

E-mail: kang97061@yahoo.com

${ }^{*}$ Contributed equally

Key words: VEGFR-2, siRNA, endothelial progenitor cell, angiogenesis recruitment of EPCs to angiogenesis represents a multistep process, including: active arrest and homing of the circulating cells within the angiogenic microvasculature; transendothelial extravasation into the interstitial space; extravascular formation of cellular clusters; creation of vascular sprouts and cellular networks; incorporation into a functional microvasculature (1). Their contribution to the formation of new blood vessels has drawn attention to their potential for therapeutic application. Recent data suggested that significant number of EPCs migrate to glioma and incorporate into vasculature with high specificity $(2,3)$. However, the molecular mechanisms responsible for EPCs angiogenesis are not fully understood. Several growth factors, including vascular endothelial growth factor, stem cell growth factor, and granulocyte-macrophage colony-stimulating factor, have been reported to be involved in the regulation of endothelial differentiation and migration to form functional vessels (4-6).

VEGFR-1 and VEGFR-2 have been considered to be the major signal transducers for both physiological and pathological angiogenesis. VEGFR-1 and VEGFR-2 have distinct functions in vascular development in embryos. VEGFR-1 knockout mice, which die around embryonic day 8.5 (E8.5), have abundant ECs, which do not assemble in functional vessels (7). In contrast, VEGFR-2 knockout mice, which also die by E8.5, lack both ECs and hematopoietic cells (8). Recent studies have emphasized the effects of VEGFR-1 on the regulation of tumor angiogenesis (9). In mature endothelial cells, VEGFR-2 functions to promote angiogenesis by regulating endothelial cell attachment, proliferation, migration and growth. However, little is understood about the role of VEGFR-1 and VEGFR-2 during glioma-associated angiogenesis of EPCs. These studies lead us to speculate that glioma cells may promote angiogenesis of EPCs via regulating the expression of VEGFR-1 or/and VEGFR-2. We therefore try to clarify the above hypothesis in the present study.

In this study we characterize that glioma cells augment angio-genesis of EPCs through up-regulating the expression of VEGFR-2 but not VEGFR-1. Then we used RNA interference method to block the endogenous VEGFR-2, and investigated the mechanism responsible for EPCs contribution to angiogenesis. We demonstrated that inhibition of migration and tube formation are detected in EPCs after RNA interference against VEGFR-2 expression. We also showed 
that VEGFR-2 siRNA inhibits MMP-9 expression and Akt and ERK phosphorylations.

\section{Materials and methods}

Cell culture. Under informed consent, mononuclear cells were isolated from human cord blood by density gradient centrifugation. Then $\mathrm{CD} 133^{+}$cells were purified by magnetic activated cell sorting columns (MACS; Miltenyi Biotech), suspended in EGM-2 MV media (Cambrex) containing endothelial basal medium (EBM), 5\% fetal bovine serum, hEGF, VEGF, hFGF-B, IGF-1, ascorbic acid, and heparin, then seeded on fibronectin-coated (Chemicon) culture dishes. After 72 h, non-adherent cells were removed. The Ethics Committee of the Nanjing Medical University approved the experiments for this study.

Human glioma U87 cells (kindly provided by Dr Jianhong Zhu, Neurosurgical Department of Huashan Hospital, Shanghai, P.R. China) were maintained in Dulbecco's modified Eagle's medium (DMEM) supplemented with $10 \%$ fetal calf serum and antibiotics (penicillin at $100 \mathrm{U} / \mathrm{ml}$ and streptomycin at $100 \mu \mathrm{g} / \mathrm{ml}$ ) in a humidified atmosphere of $5 \%$ carbon dioxide and $95 \%$ air at $37^{\circ} \mathrm{C}$. The supernatant from glioma cells was prepared by culturing cells in serum-free medium (DMEM) for $48 \mathrm{~h}$, and a centrifugal filter device (Millipore) was utilized to concentrate the supernatant from glioma cells.

Immunofluorescence staining. After 14 days of culture, cells were seeded on glass coverslips coated with collagen in 6-well plates. To detect acetylated low-density lipoprotein (Ac-LDL) uptake and ulex europaeus agglutinin-1 (UEA-1) binding, they were fixed with $4 \%$ paraformaldehyde at room temperature for $30 \mathrm{~min}$ prior to incubation with $10 \mu \mathrm{g} / \mathrm{ml}$ DiI-Ac-LDL (Molecular Probes). After fixation, cells were reacted with FITC-UEA-1 (Sigma). After the staining, cells were examined under confocal microscopy. Cells which both take up Dil-acLDL and bind FITC-UEA-1, were judged to be EPCs $(10,11)$. To detect CD31 expression, cells were incubated with goat anti-CD31 (dilution 1:100, Santa Cruz) and rabbit anti-Btubulin (dilution 1:200, Sigma) for $1 \mathrm{~h}$ at $37^{\circ} \mathrm{C}$, then incubated with Rhodamine-conjugated donkey anti-goat antibody (dilution 1:500, Santa Cruz) and FITC-conjugated goat antirabbit antibody (dilution 1:500, Invitrogen) for $30 \mathrm{~min}$ at $37^{\circ} \mathrm{C}$. Nuclei were stained with DAPI (dilution 1:1000, Molecular Probes). Images were acquired with fluorescence microscopy.

Fluorescence-activated cell sorter analysis. To assess the change of surface antigen of cells, we performed fluorescenceactivated cell sorter (FACS) analysis. Labeling was carried out using CD133/2-PE (Miltenyi Biotec) and CD31-FITC (eBioscience) in $50 \mu \mathrm{l}$ of phosphate-buffered saline (PBS) at room temperature for $30 \mathrm{~min}$. The number of positive cells was compared with the number of cells positive in staining with the immunoglobulin $\mathrm{G}$ isotype controls (BD Pharmingen) and determined with FACS flow cytometer (BD Biosciences). Three separate experiments were performed.

siRNA transfection. The sequences for VEGFR-2 silencing have been reported previously (12). The sense sequence is 5'-AAUACUUGUCGUCUGAUUCUCCAGG-3' and the anti- sense sequence is 5'-CCUGGAGAAUCAGACGACAAGUA UU-3'. The negative control siRNA: the sense sequence (5'-UUCUCCGAACGUGUCACGUTT-3'), the antisense sequence (5'-ACGUGACACGUUCGGAGAATT-3'). VEGFR-2 siRNA and negative control siRNA were both synthesized by Shanghai Genepharma Co. Inc. (Shanghai, P.R. China). EPCs at $70 \%$ confluency were transfected with VEGFR-2 siRNA, negative control siRNA and no siRNA using Lipofectamine 2000 (Invitrogen), which indicated the siRNA group, the siRNAnc group and the no siRNA group, respectively. After $4 \mathrm{~h}$, the transfection medium was removed; the cells were washed twice with PBS, and then maintained in EGM-2 MV media for $72 \mathrm{~h}$ before performing the experiments.

In vitro tube formation assay. In vitro tube formation assay was performed by plating EPCs or transfected cells $\left(5 \times 10^{3}\right.$ cells) suspended in the supernatant from glioma cells or EGM-2 MV media onto 96-well plates coated with matrigel (BD PharMingen). After $24 \mathrm{~h}$ of incubation, images of tubular structures were taken, and the total number of tube formation in three independent 200x fields for each well were calculated. Three separate experiments were performed.

In vitro cell migration assay. EPC migration was evaluated using matrigel-coated Transwell (Costar) with $8-\mu \mathrm{m}$ pore size filter. Cells were starved for $4 \mathrm{~h}$ in EBM (serum-free medium). EPCs $(200 \mu 1)$ or transfected cells suspensions in EBM $\left(10^{6} / \mathrm{ml}\right)$ were applied to the upper chamber. The supernatant from glioma cells or EGM-2 MV media was added to the lower chamber. Migration was allowed to proceed for $20 \mathrm{~h}$ at $37^{\circ} \mathrm{C}$. Cells remaining on the upper surface of the filters were carefully removed with cotton swabs. Migrated cells were stained with crystal violet and examined by light microscopy. After microscopic documentation, stained cells were extracted and measured for optical density at $560 \mathrm{~nm}$ in a microplate reader. Three separate experiments were performed.

Western blot analysis. Three hours after treatment of the supernatant from glioma cells or $72 \mathrm{~h}$ after transfection, cells were washed, harvested, and lysed in RIPA lysis buffer (Pierce) on ice for $10 \mathrm{~min}$. For phosphoprotein analysis, cell lysis buffer was supplemented with phosphatase inhibitors (1 mM sodium orthovanadate, $1 \mathrm{mM} \mathrm{NaF}$, and $10 \mathrm{mM} \mu$-glycerophosphate). The supernatants were collected and quantified for protein concentration by the Bradford assay. Equal amounts of protein per lane were separated by 5-10\% SDS-polyacrylamide gel and transferred to PVDF membrane. The membrane was blocked in 5\% skim milk for $1 \mathrm{~h}$ and then incubated with a specific antibody for $2 \mathrm{~h}$. The antibodies used for Western blot analysis in this study were: antibodies to VEGFR-1, VEGFR-2, MMP-9, Akt, phospho-Akt ${ }^{(\text {Ser473) }}$, ERK, and phospho-ERK $^{\text {(Thr202/Tyr204) }}$ (Santa Cruz). Glyceraldehyde phosphate dehydrogenase (GAPDH) was used as protein loading control. Antibody binding was detected using a chemiluminescence detection system (Pierce), according to the manufacturer's recommendations. Western blot film was digitized, and band net intensities were quantified using the Quantity One software (Bio-Rad).

Gelatin zymography. Three hours after treatment of the supernatant from glioma cells or $72 \mathrm{~h}$ after transfection, total 
A

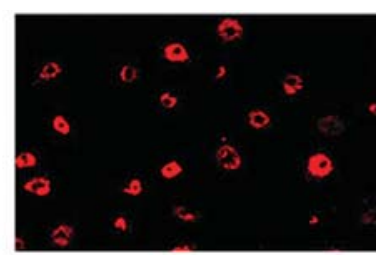

Dil-Ac-LDL

B

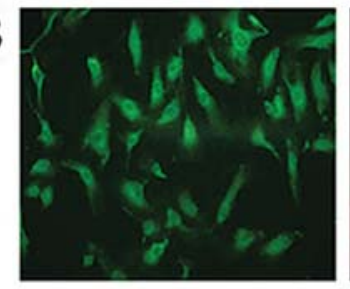

$\beta$-Tubulin
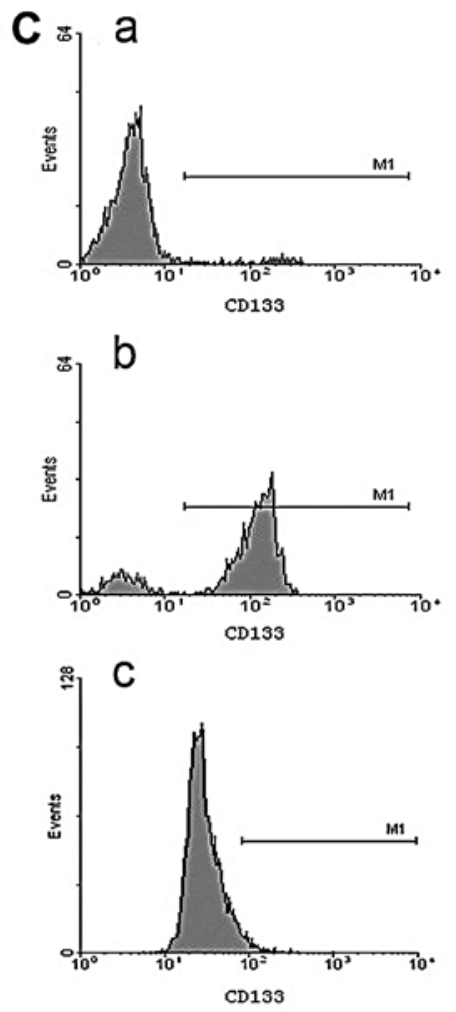

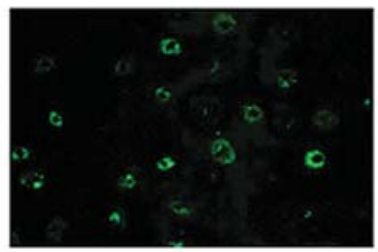

FITC-UEA-1

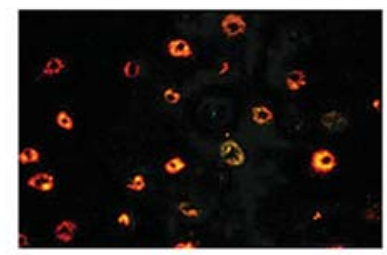

Merged

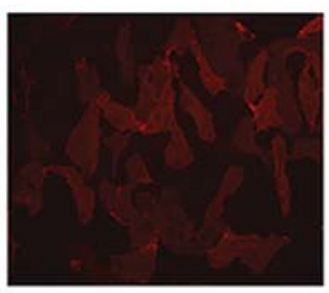

CD31

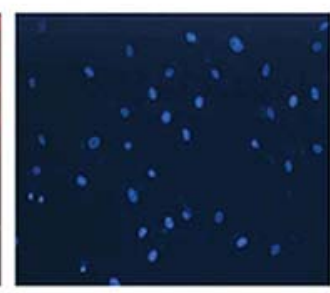

DAPI

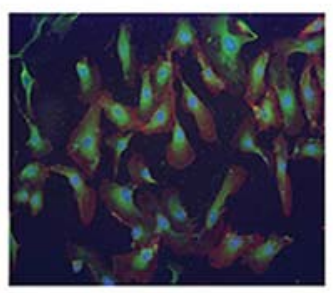

Merged
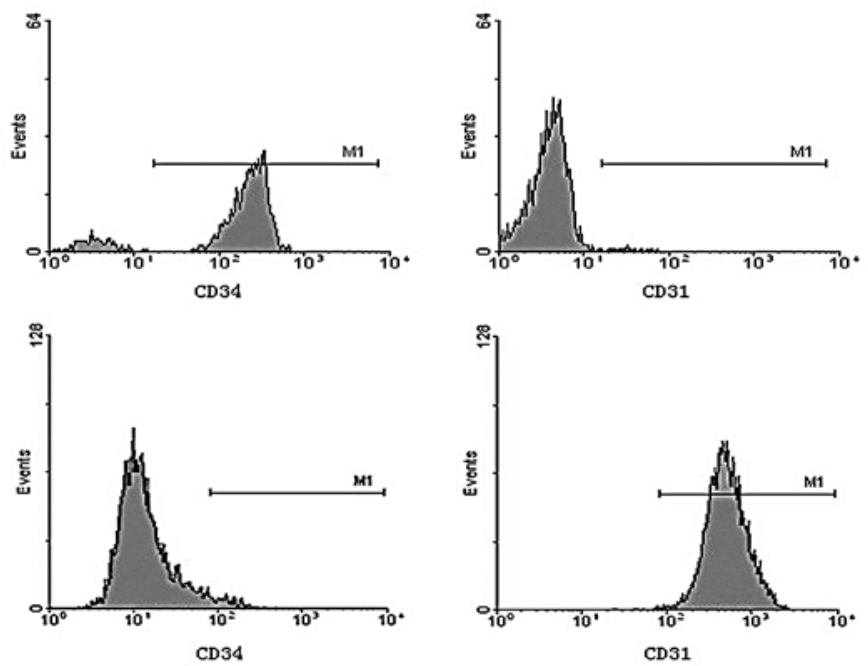

Figure 1. Phenotypic characterization of EPCs from human cord blood. (A), Two-color confocal microscopic image indicating Dil-Ac-LDL incorporation (red) and FITC-UEA-1 binding (green). Cells which both take up Dil-ac-LDL and bind FITC-UEA-1, are EPCs. (B), Three-color fluorescence microscopic image indicating B-tubulin staining (red) and CD31 staining (green). Nuclei (DAPI) are dark blue. (C), Fluorescence-activated cell sorter analysis indicating the change of surface antigen of cells (a, before CD133 immunomagnetic sorting; b, after CD133 immunomagnetic sorting; c, cultured at day 14).

cellular protein was extracted with lysis buffer (1\% NP-40 in $50 \mathrm{mM}$ Tris- $\mathrm{HCl}$ buffer, $\mathrm{pH} 8.0$ containing $150 \mathrm{mM} \mathrm{NaCl}$ ). Equal amounts of protein from the treatment groups were electrophoresed on a gelatin containing $8 \%$ SDS-polyacrylamide gel. Following electrophoresis, the gel was incubated in the washing buffer $(50 \mathrm{mM}$ Tris- $\mathrm{HCl}, \mathrm{pH} 7.5,2.5 \%$ Triton $\mathrm{X}-100)$. Then the gel was incubated in reaction buffer $(50 \mathrm{mM}$ Tris-HCl, $\mathrm{pH} 7.5$, containing $5 \mathrm{mM} \mathrm{CaCl} 2,150 \mathrm{mM} \mathrm{NaCl}$, $0.02 \% \mathrm{NaN}_{3}$ ) at $37^{\circ} \mathrm{C}$. Twenty-four hours later the gel was stained with $0.05 \%$ Coomassie Blue. Gels were visualized by Gel Doc ${ }^{\mathrm{TM}}$ XR gel documentation system (Bio-Rad).

Statistical analysis. Data were analyzed with Stata 7.0. Statistical significance $(\mathrm{P}<0.05)$ was determined by One-Way
ANOVA and independent sample two-tailed t-tests followed by assessment of differences.

\section{Results}

EPCs culture and identification. After 7 days of culture, the $\mathrm{CD}_{133^{+}}$cells isolated from human cord blood mononuclear cells exhibited small colonial morphology; then, the colonies expanded, confluenced, and displayed a typical 'cobblestone' morphology. More than 90\% attached cells took up Dil-acLDL and bound FITC-UEA-1 (Fig. 1A), and were judged to be EPCs. The endothelial phenotype of expanded EPCs was further characterized by expression of endothelial markers such as CD31 (Fig. 1B). Compared with the original, cell 


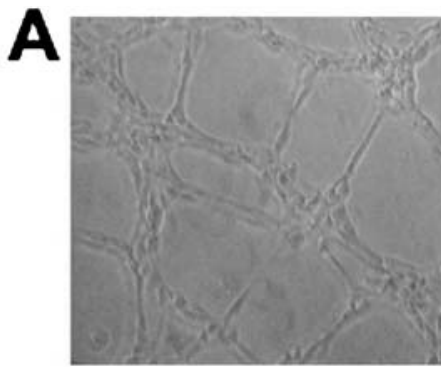

U87

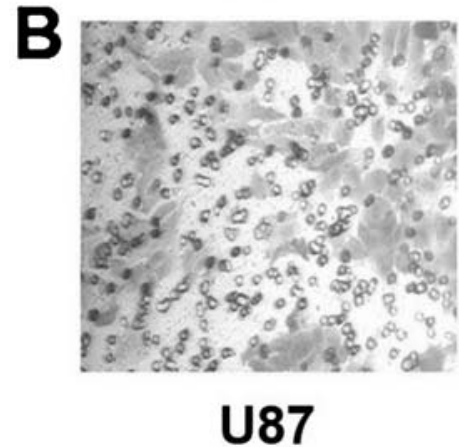

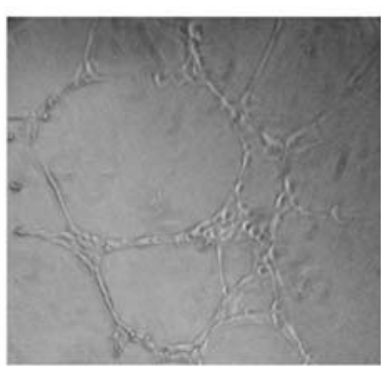

DMEM

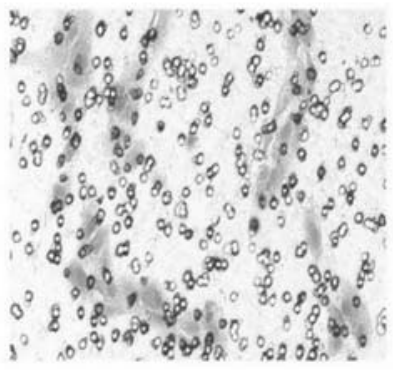

DMEM
Figure 2. The supernatant from glioma cells increases EPC tube formation on matrigel and migration of EPCs. (A), Tubes were visualized by phase contrast microscopy at a magnification of $\mathrm{x} 200$. The supernatant from glioma cells significantly induces formation of tube structures, compared with DMEM $(40.7 \pm 3.3$ versus $21 \pm 2.3, P<0.05)$. (B), Migrated cells were stained with crystal violet and examined with light microscopy at a magnification of $\mathrm{x} 200$. The OD values were $0.60 \pm 0.04,0.27 \pm 0.02$, respectively for the supernatant from glioma cells and DMEM $(\mathrm{P}<0.05)$.

markers CD133 and CD34 decreased from $86.04 \pm 3.36 \%$ to $2.96 \pm 0.45 \%$ and from $90.88 \pm 2.86 \%$ to $2.99 \pm 0.11 \%$, respectively, while CD31 increased from $1.12 \pm 0.04 \%$ to $99.88 \pm 0.04 \%$ (Fig. 1C). EPCs had high potential of proliferation and expanded for at least 15 PDs without obvious signs of senescence. Based on our data, EPCs we cultured were similar to late EPCs, which were first proposed by Hur et al (13).

The supernatant from glioma cells increases EPC tube formation on matrigel and migration. To explore the effect of the supernatant from glioma cells on angiogenic function of EPCs, we performed tube formation on matrigel and migration assay. In the absence of the supernatant from glioma cells, capillary morphogenesis of EPCs was clearly induced, compared with DMEM $(40.7 \pm 3.3$ versus $21 \pm 2.3, \mathrm{P}<0.05)$ (Fig. 2A). In the migration assay, the OD values were $0.60 \pm 0.04$ and $0.27 \pm 0.02$, respectively for the supernatant from glioma cells and DMEM $(\mathrm{P}<0.05)$ (Fig. 2B). These results suggest that the supernatant from glioma cells augmented the ability of EPCs angiogenesis.

The supernatant from glioma cells up-regulates VEGFR-2 and MMP-9 expression of EPCs not VEGFR-1. In order to examine whether VEGFR-1 and VEGFR-2 are involved in EPC angiogenesis regulated by the supernatant from glioma cells, Western blot analysis was performed. A strong induction of VEGFR-2 protein was observed when EPCs were cultured with the supernatant from glioma cells. VEGFR-2 expression

\section{MMP-9 activity}

MMP-9 protein

VEGFR-2

\section{VEGFR-1}

\section{GAPDH}
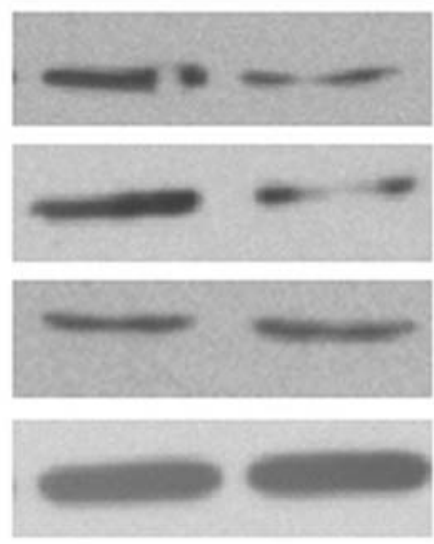

U87

DMEM

Figure 3. The supernatant from glioma cells up-regulates expression of VEGFR-2 and MMP-9, not VEGFR-1 in EPCs. Effects of the supernatant from glioma cells on VEGFR-1, VEGFR-2 and MMP-9 expression were determined by Western blot analysis. The quantitative data show relative protein levels of VEGFR-1, VEGFR-2 and MMP-9 (normalized to GAPDH level) in the no siRNA group. Cell lysates were evaluated for MMP-9 activity by zymography analysis and protein expression by Western blot analysis as described in Materials and methods.

significantly increased by $2.6 \pm 0.1$-fold compared with the treatment of DMEM, while expression levels of VEGFR-1 were almost unchanged (Fig. 3). Additionally, we investigated the MMP-9 activity and expression of EPCs after the treatment of the supernatant from glioma cells by gelatin zymography and Western blot analysis. As shown in Fig. 3, the induction of MMP-9 expression was detected (2.5 \pm 0.2 -fold) with the regulation of the supernatant from glioma cells. A similar change was observed in MMP-9 activity. These results indicated that the supernatant from glioma cells enhanced VEGFR-2 and MMP-9 expression of EPCs, not VEGFR-1.

Reduction of VEGFR-2 expression in EPCs by siRNA. Seventytwo hours after transfection, the cell lysates were analyzed by Western blot analysis for VEGFR-2 expression. As shown in Fig. 4, VEGFR-2 duplexes triggered a marked decrease in the protein levels for the target proteins in EPCs, with minimal effect on the expression of VEGFR-1 and GAPDH. These results indicated that VEGFR-2 siRNA effectively and specifically down-regulated VEGFR-2 protein expression in EPCs.

VEGFR-2 siRNA inhibits EPC tube formation on matrigel and the migration. To evaluate the impact of VEGFR-2 knock-down on EPC tube formation, we employed a matrigel tube formation assay using the VEGFR-2 siRNA transfected EPCs. When compared with the siRNAnc group and the no siRNA group, VEGFR-2 siRNA transfected cells lost the ability to form tubular networks on matrigel, failed to form tube-like networks (Fig. 5A), suggesting that EPCs tube formation on matrigel is regulated by the expression of VEGFR-2. As observed in the migration assays, we found that the migration capacity of the cells transfected with 
VEGFR-2

VEGFR-1
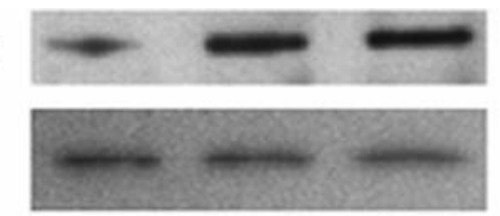

GAPDH

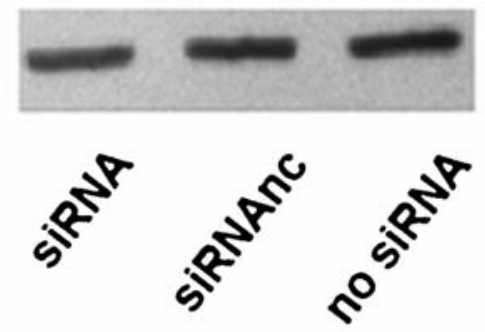

Figure 4. VEGFR-2 siRNA down-regulates expression of VEGFR-2 in EPCs. EPCs were treated with VEGFR-2 siRNA, siRNAnc or no siRNA as described in Materials and methods. VEGFR-2, VEGFR-1 and GAPDH expressions were determined by Western blot analysis. The quantitative data show relative protein levels of VEGFR-2, VEGFR-1 (normalized to GAPDH level) in the no siRNA group.

VEGFR-2 siRNA was significantly decreased compared with that of the cells transfected with control siRNA (Fig. 5B). The OD values were $0.24 \pm 0.06,0.56 \pm 0.06$ and $0.59 \pm 0.08$, respectively for the VEGFR-2 siRNA, siRNAnc and no siRNA group $(\mathrm{P}<0.05)$. These experiments demonstrated that VEGFR-2 was required for EPC tube formation and migration.

VEGFR-2 siRNA inhibits MMP-9 protein expression and MMP-9 activity of EPCs. In order to explore the role of
VEGFR-2 in the MMP-9 activity and expression of EPCs, we used gelatin zymography and Western blot analysis, respectively. MMP-9 activity of VEGFR-2 siRNA was significantly decreased $72 \mathrm{~h}$ after transfection (Fig. 6A). Consistently, Western blot analysis demonstrated a pronounced reduction in MMP-9 protein expression as compared to the siRNAnc group and the no siRNA group, suggesting that decreased activity of MMP-9 in VEGFR-2 siRNA transfected cells is due to an inhibition of protein expression. Thus, VEGFR-2 appeared to be an important factor in the regulation of MMP-9 expression.

VEGFR-2 inhibits the Akt and ERK signaling pathways in EPCs. To investigate whether the Akt and ERK pathways were down-regulated by VEGFR-2 siRNA transfected EPCs, phosphorylations of Akt and ERK were measured (Fig. 6B). Activation of Akt by VEGFR-2 siRNA was significantly reduced by $\sim 50 \%$ compared with the siRNAnc group and the no siRNA group, while almost a $30 \%$ reduction of phosphorylation of ERK was detected. No obvious changes were observed for total Akt and ERK protein expressions. The results indicated that the Akt and ERK pathways were major signaling pathways in VEGFR-2 regulation.

\section{Discussion}

In this study, we evaluated the function of glioma cells in EPC angiogenesis. Our data show that the supernatant from glioma cells can enhance EPC tube formation and migration via up-regulating VEGFR-2 expression, not VEGFR-1. Furthermore, VEGFR-2 siRNA silences endogenous VEGFR-2

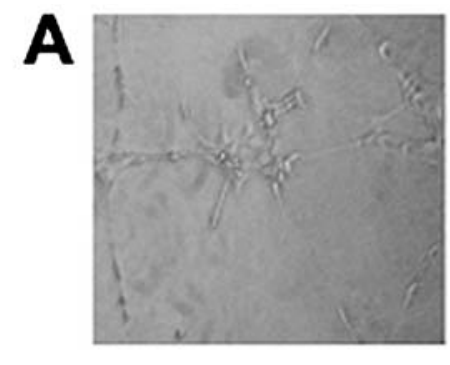

SIRNA

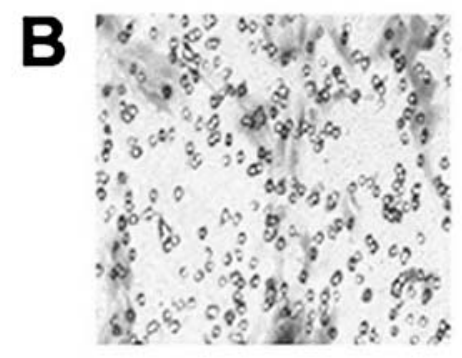

SIRNA

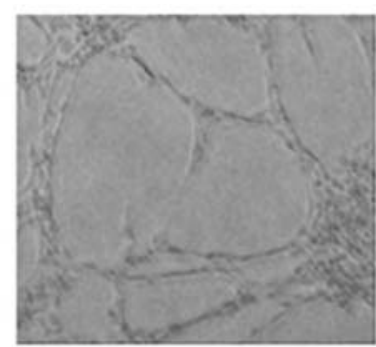

SiRNAnc

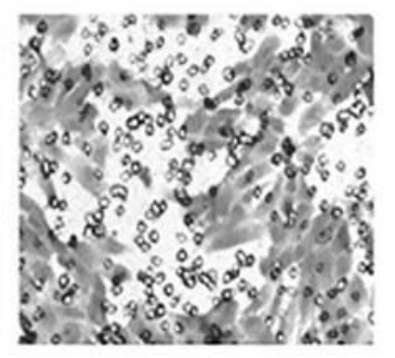

SiRNAnc

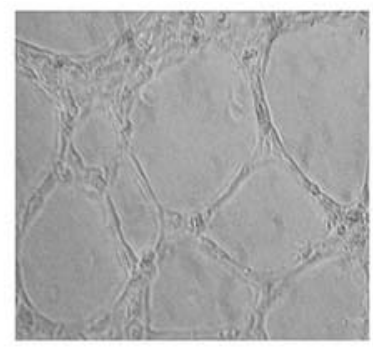

no SIRNA

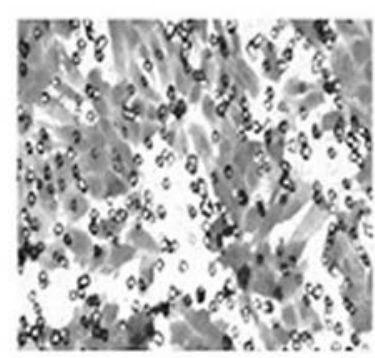

no SIRNA

Figure 5. VEGFR-2 siRNA inhibits EPC tube formation on matrigel and migration of EPCs. (A), Tubes were visualized by phase contrast microscopy at a magnification of $x 200$. VEGFR-2 siRNA transfected cells lost the ability to form tubular networks on matrigel, and the number of tube are $15.0 \pm 6.0$ ( $<<0.05)$, compared with the siRNAnc group (45.7 \pm 4.9$)$ and the no siRNA group (40.3 \pm 4.2$)$. (B), Migrated cells were stained with crystal violet and examined with light microscopy at a magnification of $x 200$. The OD values were $0.24 \pm 0.06,0.56 \pm 0.06,0.59 \pm 0.08$, respectively for the VEGFR-2 siRNA, siRNAnc and no siRNA group $(\mathrm{P}<0.05)$. 
A

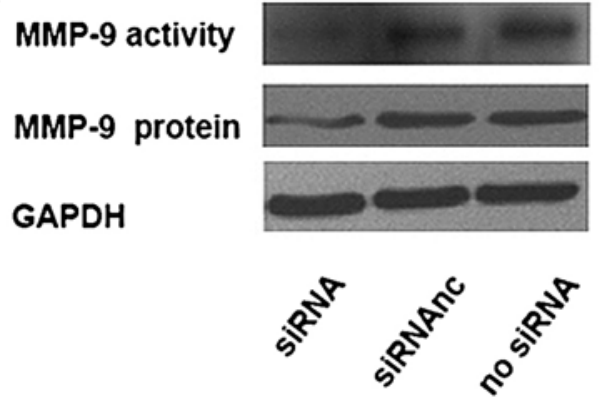

B

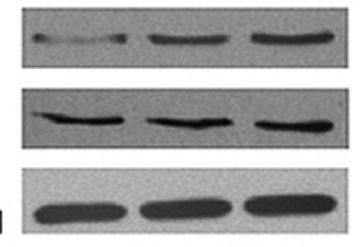

P-ERK
ERK
GAPDH

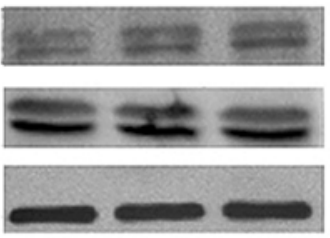

,

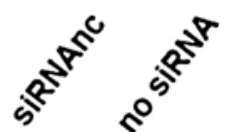

Figure 6. VEGFR-2 siRNA inhibits activity and expression of MMP-9 through Akt and ERK pathways in EPCs. (A), Cell lysates were evaluated for MMP-9 activity by zymography analysis and protein expression by Western blot analysis as described in Materials and methods. (B), Effects of VEGFR-2 siRNA on MMP-9, Akt, phospho-Akt, ERK and phospho-ERK expression were determined by Western blot analysis. The quantitative data show relative protein levels of MMP-9, Akt, phospho-Akt, ERK and phospho-ERK (normalized to GAPDH level) in the no siRNA group.

expression in EPCs, and inhibits EPC angiogenesis by downregulating the MMP-9, Akt and ERK pathways.

Although a great deal is known about the mechanisms and key regulators of VEGFR-1 and VEGFR-2 involved in angiogenesis, reports on the effect of VEGFR-1 and VEGFR-2 on glioma-associated angiogenesis of EPCs are few. VEGFR-1 and VEGFR-2 are key proteins modulating vascular development during angiogenesis. VEGFR-1 has a structure very similar to VEGFR-2 and binds to VEGF with a higher affinity than VEGFR-2. The role of VEGFR-1 has been debated and its exact function is still unclear (9). On the contrary, VEGFR-2 is a major positive signal transducer and it shows a higher kinase activity than VEGFR-1. Based on analyses of protein level, our data reveal that the supernatant from glioma cells up-regulates EPC angiogenesis via induction of VEGFR-2 expression, while no significant change in VEGFR-1 protein expression was observed.

In order to investigate the further mechanism responsible for glioma cell contribution to EPCs angiogenesis, we use RNA interference method to block the endogenous VEGFR-2. Most of the studies on the involvement of VEGFR-2 during angiogenesis were either carried out in the presence of serum or growth factors, which are inducers of VEGFR-2, or in the presence of VEGFR-2 inhibitors. The use of natural or synthetic inhibitors against VEGFR-2 might not completely explain the role of VEGFR-2, as these inhibitors can also affect various other components of angiogenic process. This is evidenced by the report where SU5416 inhibited angiogenesis by binding to VEGFR-1, VEGFR-2 and EGF receptors (14). The advantages of RNA interference over other modalities include its high specificity (in some cases, a single point mutation can abolish silencing effect), versatility (interfering RNA can be designed against virtually any gene), and efficiency (in many cases, genes can be silenced by over $90 \%)(15,16)$. Thus, the relatively new technique of RNA interference with short double-stranded RNA molecules that are complementary to the target mRNA is a potentially powerful tool to silence endogenous VEGFR-2 and evaluate its function. We chose the small interfering RNAs technique to explore how VEGFR-2 exerts its activity in EPCs. VEGFR-2 protein expression is significantly decreased $72 \mathrm{~h}$ after transfection of VEGFR-2 siRNA. In contrast, non-silencing RNA duplexes have no effect on VEGFR-2 expression. Expression levels of VEGFR-1 and GAPDH are almost unchanged in the two different siRNA groups, indicating that the transfection procedure has no effect on the expression of these genes. The functional consequences of silencing VEGFR-2 expression in EPCs through VEGFR-2 siRNA were investigated. EPCs with diminished VEGFR-2 expression are less angiogenic and migrative as compared to the siRNAnc and no siRNA group.

Moreover, in our study, the supernatant from glioma cells obviously enhances the MMP-9 activity and expression of EPCs. MMP-9, a key enzyme that functions to promote angiogenesis by regulating endothelial cell attachment, proliferation, migration and growth (17-19). MMP-9, which leads to the release of soluble KIT ligand, promotes the proliferation and motility within the bone marrow microenvironment, thereby laying the framework for EPC mobilization to the peripheral circulation, and homing to the vasculature $(20,21)$. It is observed that reducing the expression of VEGFR-2 inhibits the activity and expression of MMP-9. Thus, our data indicate that VEGFR-2 siRNA inhibits EPCs angiogenesis via down-regulating the activity and expression of MMP-9.

Additionally, the Akt and ERK pathways have been documented to contribute to signal transduction pathways in regulation of VEGFR-2 and MMP-9 (12,22-24). Therefore, we hypothesize that VEGFR-2 is a key modulator of angiogenesis by modulating Akt and ERK pathways in EPCs. As is well-known, the Akt and ERK pathways are involved in angiogenesis. Akt, also known as protein kinase B (PKB), regulates essential cellular functions such as migration, proliferation, differentiation, apoptosis, and metabolism. In endothelial cells, the majority of growth factor-induced angiogenic responses are mediated by the activation of the Akt signaling cascade (25). In HUVEC angiogenesis, VEGFR-2 protein expression is associated with phosphorylation of Akt (24). Additionally, Hur et al (26) have reported that Akt plays a critical role in the homing of EPCs to the ischemic muscle through increased attachment and transendothelial migration, and its modulation enhances the homing of EPCs and new vessel formation in target organs. ERK is a member of the family of mitogen-activated protein kinases and has been demonstrated to play an important role in transmitting extracellular signals into various cellular 
responses such as proliferation and migration. VEGFR-2 acts via the ERK pathway which is known to lead to endothelial cell modulation $(27,28)$. Consistent with these premises, VEGFR-2 siRNA blocks both the Akt and ERK pathways, although inhibition of other pathways by VEGFR-2 siRNA cannot be ruled out. Our data strongly show that the Akt and ERK pathways are both involved in VEGFR-2 signal transduction, however, the further mechanism is still under investigation.

In summary, we have demonstrated that glioma cells enhance EPCs angiogenesis via VEGFR-2, not VEGFR-1. Further, VEGFR-2 siRNA effectively and specifically reduces the expression of VEGFR-2 in EPCs. Down-regulation of VEGFR-2 inhibits the MMP-9, Akt and ERK pathways in EPC angiogenesis. Therefore, the results of the present study suggest that the modulation of the mechanism responsible for EPC-mediated glioma angiogenesis may be used as a therapeutic strategy to affect pathological angiogenesis and thus warrants further investigation.

\section{Acknowledgements}

This work was supported by the Medicine and Technology Development Foundation of Jiangsu Health Department (H200510) and Jiangsu Province Medical major Talent Program (RC2007061).

\section{References}

1. Ribatti D: The involvement of endothelial progenitor cells in tumor angiogenesis. J Cell Mol Med 8: 294-300, 2004.

2. Ferrari N, Glod J, Lee J, Kobiler D and Fine HA: Bone marrowderived, endothelial progenitor-like cells as angiogenesisselective gene-targeting vectors. Gene Ther 10: 647-656, 2003.

3. Moore XL, Lu J, Sun L, Zhu CJ, Tan P and Wong MC: Endothelial progenitor cells' 'homing' specificity to brain tumors. Gene Ther 11: 811-818, 2004.

4. Asahara T, Masuda H, Takahashi T, Kalka C, Pastore C, Silver M, Kearne M, Magner M and Isner JM: Bone marrow origin of endothelial progenitor cells responsible for postnatal vasculogenesis in physiological and pathological neovascularization. Circ Res 85: 221-228, 1999.

5. Asahara T, Takahashi T, Masuda H, Kalka C, Chen D, Iwaguro H, Inai $\mathrm{Y}$, Silver $\mathrm{M}$ and Isner JM: VEGF contributes to postnatal neovascularization by mobilizing bone marrow-derived endothelial progenitor cells. EMBO J 18: 3964-3972, 1999.

6. Gehling UM, Ergun S, Schumacher U, Wagener C, Pantel K, Otte M, Schuch G, Schafhausen P, Mende T, Kilic N, Kluge K, Schafer B, Hossfeld DK and Fiedler W: In vitro differentiation of endothelial cells from AC133-positive progenitor cells. Blood 95: 3106-3112, 2000.

7. Fong GH, Rossant J, Gertsenstein M and Breitman ML: Role of the Flt-1 receptor tyrosine kinase in regulating the assembly of vascular endothelium. Nature 376: 66-70, 1995.

8. Shalaby F, Rossant J, Yamaguchi TP, Gertsenstein M, Wu XF, Breitman ML and Schuh AC: Failure of blood-island formation and vasculogenesis in Flk-1-deficient mice. Nature 376: 62-66, 1995.

9. Shibuya M: Differential roles of vascular endothelial growth factor receptor- 1 and receptor- 2 in angiogenesis. J Biochem Mol Biol 39: 469-478, 2006.

10. Kalka C, Masuda H, Takahashi T, Kalka-Moll WM, Silver M, Kearney M, Li T, Isner JM and Asahara T: Transplantation of ex vivo expanded endothelial progenitor cells for therapeutic neovascularization. Proc Natl Acad Sci USA 97: 3422-3427, 2000.

11. Vasa M, Fichtlscherer S, Aicher A, Adler K, Urbich C, Martin H, Zeiher AM and Dimmeler S: Number and migratory activity of circulating endothelial progenitor cells inversely correlate with risk factors for coronary artery disease. Circ Res 89: E1-E7, 2001 .
12. Zimman A, Mouillesseaux KP, Le T, Gharavi NM, Ryvkin A, Graeber TG, Chen TT, Watson AD and Berliner JA: Vascular endothelial growth factor receptor 2 plays a role in the activation of aortic endothelial cells by oxidized phospholipids. Arterioscler Thromb Vasc Biol 27: 332-338, 2007.

13. Hur J, Yoon CH, Kim HS, Choi JH, Kang HJ, Hwang KK, Oh BH, Lee MM and Park YB: Characterization of two types of endothelial progenitor cells and their different contributions to neovasculogenesis. Arterioscler Thromb Vasc Biol 24: 288-293, 2004.

14. Haspel HC, Scicli GM, McMahon G and Scicli AG: Inhibition of vascular endothelial growth factor-associated tyrosine kinase activity with SU5416 blocks sprouting in the microvascular endothelial cell spheroid model of angiogenesis. Microvasc Res 63: 304-315, 2002.

15. Aigner A: Gene silencing through RNA interference (RNAi) in vivo: strategies based on the direct application of siRNAs. J Biotechnol 124: 12-25, 2006.

16. Li CX, Parker A, Menocal E, Xiang S, Borodyansky L and Fruehauf JH: Delivery of RNA interference. Cell Cycle 5: 2103-2109, 2006.

17. Bergers G, Brekken R, McMahon G, Vu TH, Itoh T, Tamaki K, Tanzawa K, Thorpe P, Itohara S, Werb Z and Hanahan D. Matrix metalloproteinase-9 triggers the angiogenic switch during carcinogenesis. Nat Cell Biol 2: 737-744, 2000

18. Gu Z, Kaul M, Yan B, Kridel SJ, Cui J, Strongin A, Smith JW, Liddington RC and Lipton SA: S-nitrosylation of matrix metalloproteinases: signaling pathway to neuronal cell death. Science 297: 1186-1190, 2002.

19. Li A, Dubey S, Varney ML, Dave BJ and Singh RK: IL-8 directly enhanced endothelial cell survival, proliferation, and matrix metalloproteinases production and regulated angiogenesis. J Immunol 170: 3369-3376, 2003.

20. Engsig MT, Chen QJ, Vu TH, Pedersen AC, Therkidsen B, Lund LR, Henriksen K, Lenhard T, Foged NT, Werb Z and Delaisse JM: Matrix metalloproteinase 9 and vascular endothelial growth factor are essential for osteoclast recruitment into developing long bones. J Cell Biol 151: 879-889, 2000.

21. Vu TH and Werb Z: Matrix metalloproteinases: effectors of development and normal physiology. Genes Dev 14: 2123-2133, 2000.

22. Byun HJ, Hong IK, Kim E, Jin YJ, Jeoung DI, Hahn JH, Kim YM, Park SH and Lee H: A splice variant of CD99 increases motility and MMP-9 expression of human breast cancer cells through the AKT-, ERK-, and JNK-dependent AP-1 activation signaling pathways. J Biol Chem 281: 34833-34847, 2006.

23. Kim CW, Son KN, Choi SY and Kim J: Human lactoferrin upregulates expression of KDR/Flk-1 and stimulates VEGF-Amediated endothelial cell proliferation and migration. FEBS Lett 580: 4332-4336, 2006.

24. Xiao D, Li M, Herman-Antosiewicz A, Antosiewicz J, Xiao H, Lew KL, Zeng Y, Marynowski SW and Singh SV: Diallyl trisulfide inhibits angiogenic features of human umbilical vein endothelial cells by causing Akt inactivation and down-regulation of VEGF and VEGFR-2. Nutr Cancer 55: 94-107, 2006.

25. Shiojima I and Walsh K: Role of Akt signaling in vascular homeostasis and angiogenesis. Circ Res 90: 1243-1250, 2002.

26. Hur J, Yoon CH, Lee CS, Kim TY, Oh IY, Park KW, Kim JH, Lee HS, Kang HJ, Chae IH, Oh BH, Park YB and Kim HS: Akt is a key modulator of endothelial progenitor cell trafficking in ischemic muscle. Stem Cells 25: 1769-1778, 2007.

27. Cho CH, Lee CS, Chang M, Jang IH, Kim SJ, Hwang I, Ryu SH, Lee $\mathrm{CO}$ and Koh GY: Localization of VEGFR-2 and PLD2 in endothelial caveolae is involved in VEGF-induced phosphorylation of MEK and ERK. Am J Physiol Heart Circ Physiol 286: H1881-H1888, 2004.

28. Guo DQ, Wu LW, Dunbar JD, Ozes ON, Mayo LD, Kessler KM, Gustin JA, Baerwald MR, Jaffe EA, Warren RS and Donner DB: Tumor necrosis factor employs a protein-tyrosine phosphatase to inhibit activation of KDR and vascular endothelial cell growth factor-induced endothelial cell proliferation. J Biol Chem 275: 11216-11221, 2000 